\title{
Avoidance of ischemic complications after resection of a brain lesion based on intraoperative real-time recognition of the vasculature using laser speckle flow imaging
}

\author{
Makoto Ideguchi, MD, ${ }^{1}$ Koji Kajiwara, MD, ${ }^{2}$ Koichi Yoshikawa, MD, ${ }^{1}$ Hisaharu Goto, MD, ${ }^{1}$ \\ Kazutaka Sugimoto, MD, ${ }^{1}$ Takao Inoue, PhD, ${ }^{1}$ Sadahiro Nomura, MD, ${ }^{1}$ and Michiyasu Suzuki, MD' \\ 1Department of Neurosurgery, Yamaguchi University Graduate School of Medicine; and 2Department of Neurosurgery, \\ Ube-nishi Rehabilitation Hospital, Ube, Japan
}

\begin{abstract}
OBJECTIVE To avoid ischemic complications, it is important to consider the arteries in resection planning for lesions such as a vascular intraparenchymal tumor and arteriovenous malformation. Here, the clinical application of laser speckle flow imaging (LSFI) as a complementary method for the management of mass lesion-related arteries during surgery was evaluated.
\end{abstract}

METHODS LSFI was performed in 12 patients with mass lesion-related arteries and brain tumor or arteriovenous malformation. The portable LSFI device was centered over the surgical field, and the relative cerebral blood flow (CBF) before and after the temporary interruption of the arteries was measured through continuous recording. CBF fluctuations permitted the classification of 3 kinds of artery - a feeding artery (FA), a "passing through" artery (PA), and a combined $F A$ and $P A(F A+P A)-$ based on decreased relative $C B F$ in the inner resection area and unchanged $C B F$ in the surrounding area (FA), unchanged $C B F$ in the inner area and decreased $C B F$ in the surrounding area (PA), or decreased $C B F$ in both areas (FA+PA). This information allowed the appropriate management of these arteries and avoidance of postoperative ischemic complications.

RESULTS Good visualization of CBF in the surgical field and relative CBF measurements in the regions of interest were achieved in real time with excellent spatiotemporal resolution. In 11 patients (92\%) and 20 regions of interest, a decline in CBF was observed after temporary interruption of the $F A(n=8), P A(n=2)$, and FA+PA $(n=2)$ types. There was a significant average reduction in CBF of $15.3 \% \pm 29.0 \%$. There were no ischemic complications, and only 1 patient had a postoperative ischemic lesion caused by resection through an artery that could not be viewed by LSFI due to a positional problem.

CONCLUSIONS LSFI permits noninvasive and rapid intraoperative real-time recognition of mass lesion-related vasculature. This information can be used to avoid ischemic complications as a procedure complementary to neurophysiological monitoring.

http://thejns.org/doi/abs/10.3171/2016.1.JNS152067

KEY WORDS laser speckle flow imaging; cerebral blood flow; vascular lesion surgery; recognition of vasculature; intraoperative monitoring; diagnostic and operative techniques

$\mathrm{I}$ $\mathrm{N}$ surgery for a vascular tumor or arteriovenous malformation (AVM), it is important to avoid complications associated with an ischemic lesion formed by damage to the arteries. Indocyanine green (ICG) videoangiography or intraoperative digital subtraction angiography (DSA) can be used to evaluate vascular patency during bypass ${ }^{30,32}$ or aneurysm ${ }^{5,25}$ surgery, but these procedures do not visualize relative microcirculatory flow or tissue perfusion. Laser speckle flow imaging (LSFI) was developed in the 1980s for monitoring blood flow in the retina ${ }^{4,11}$ and is now commonly used to monitor changes in cerebral blood flow $(\mathrm{CBF})^{1,7,18-20,24,26,29,33}$ during functional activation, ${ }^{6,8,10,16}$ cortical spreading depression, ${ }^{2,27}$ and hypothermia. ${ }^{17}$ LSFI has been used in extracranial-intracranial bypass surgery since 2009. $9,13,14$

We have shown that LSFI measurement of the intraoperative decrease in CBF caused by internal carotid artery interruption is almost equal to that during preoperative balloon test occlusion measured by iodoamphetamine SPECT, and the intraoperative increase in CBF by superficial tem-

ABBREVIATIONS AVM = arteriovenous malformation; $\mathrm{CBF}=$ cerebral blood flow; $\mathrm{DSA}=$ digital subtraction angiography; $\mathrm{FA}=\mathrm{feeding}$ artery; $\mathrm{FA}+\mathrm{PA}=\mathrm{combined} \mathrm{FA}$ and $\mathrm{PA}$; iCBF = intensity of CBF; ICG = indocyanine green; LSFI = laser speckle flow imaging; PA = "passing through" artery; ROI = region of interest.

SUBMITTED September 1, 2015. ACCEPTED January 12, 2016.

INCLUDE WHEN CITING Published online April 1, 2016; DOI: 10.3171/2016.1.JNS152067. 
poral artery-middle cerebral artery anastomosis and the postoperative increase in CBF are moderately correlated. ${ }^{22}$ Thus, using LSFI intraoperatively permits the recognition of the perfusion area of a temporarily interrupted artery in real time. Moreover, a comparison of CBF fluctuation between the planned resection area and surrounding normal surface area might allow the classification of an artery as a feeding artery, a "passing through" artery that is just perforating the mass lesion, or both. Appropriate management of each artery type might then reduce ischemic complications. In this study, we investigated the utility of intraoperative LSFI for measuring CBF and avoiding postoperative ischemic complications during tumor or AVM surgery.

\section{Methods \\ Patients}

From May 2011 to April 2014, intraoperative CBF was measured using LSFI in 12 patients with a brain tumor (n $=7)$ or AVM $(n=5)$ at Yamaguchi University Hospital. The patients included 8 males and 4 females, and they had an average age of $61.9 \pm 16.9$ years (range 3-75 years). The study protocol, including the use of a new device, was approved by the institutional review board at Yamaguchi University and conformed to the Declaration of Helsinki. The patients and/or their families agreed to participate in this study after being adequately informed of the aims, methods, and anticipated benefits, as well as the potential risks and discomforts. All patients had the right to withdraw from the study at any time.

\section{LSFI During Surgery}

We previously described our use of LSFI during surgery. ${ }^{22}$ Briefly, the LSFI system (Omegazone OZ-1; Omegawave Inc.) consisted of a laser emitter, infrared filter, charge-coupled device camera, and analysis software. The charge-coupled device camera was mounted on a surgical microscope (OPMI Pentero; Carl Zeiss Meditec) and captured random speckle patterns from laser-illuminated red blood cells on the brain surface. The velocity of red blood cell movement was converted into a 2D CBF map on a laptop computer that was placed at the surgeon's elbow. $\mathrm{CBF}$ was quantified and expressed as the intensity (iCBF) with arbitrary units. The surgeon can easily see the 2D CBF map on the laptop monitor placed at the surgeon's elbow and can also check real-time fluctuations on the timeline chart.

\section{LSFI Protocol}

Four to 8 regions of interest (ROIs) covering the entire exposed surface area were selected without inclusion of the large arteries or veins. A total of 92 ROIs were measured, with 7.6 ROIs per case on average. The average iCBF from the ROIs was determined. The rate of change in $\mathrm{iCBF}$ was calculated as follows: (preprocedure $\mathrm{iCBF}$ - postprocedure $\mathrm{iCBF}$ )/preprocedure iCBF. We evaluated iCBF before and after interrupting an artery related to the planned resection area using a temporary clip. The schema in Fig. 1 shows the principles underlying the classification of an artery in the resection area. Lesion borders were defined using a neuronavigation system (StealthStation
A c. feeding and passing through artery



B

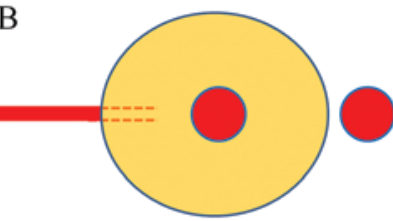

C

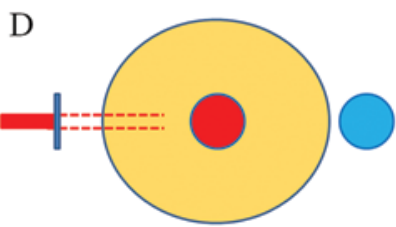

$\mathrm{E}$

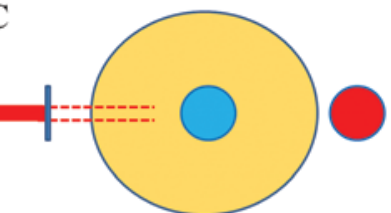

FIG. 1. Principles of artery classification based on CBF fluctuations after the temporary interruption of lesion-related arteries in the inner and outer regions of the planned resection area. A small red circle indicates normal CBF, and a small blue circle indicates decreased CBF in the ROI. A: Schema showing the 3 types of arteries categorized by CBF fluctuation. B: Normal CBF before artery interruption. C: FA based on decreased $\mathrm{CBF}$ in the inner region of the resection area and unchanged $\mathrm{CBF}$ in the surrounding area. D: PA based on unchanged CBF in the inner region and decreased $C B F$ in the surrounding area. $E$ : FA and PA based on decreased CBF in the inner and outer regions of the resection area.

S7; Medtronic Inc.). Three types of artery were defined: a feeding artery (FA) that fed the resection area only; a "passing through" artery (PA) that did not feed the resection area but perfused the surrounding normal area; and a combined FA+PA artery that possessed the features of an FA and PA (Fig. 1A). A lesion-related FA+PA artery passes through the resection planning area, but when the branching small arteries from this PA feed the resection planning area, then the PA also becomes an FA. Thus, the artery was classified as a FA+PA, as shown in Fig. 1A and E. Normal iCBF indicates normal perfusion (red circle) in the inner region of the resection area and surrounding normal region (Fig. 1B). After interrupting an artery, the artery was defined as FA if iCBF decreased (blue circle) in the inner region of the resection area and remained unchanged in the surrounding area (Fig. 1C), the artery was defined as PA if $\mathrm{iCBF}$ was unchanged (blue circle) in the inner region and decreased in the surrounding area (Fig. 1D), and the artery was defined as FA+PA if iCBF decreased in the inner and outer regions of the resection area (Fig. 1E). Usually, 10 to 15 minutes are required for complete classification of the lesion-related arteries in each patient. Based on this classification, arteries were managed appropriately to avoid postoperative ischemic complications. Specifically, an FA can be sacrificed while 
TABLE 1. Clinical characteristics of 12 patients monitored by LSFI during surgery*

\begin{tabular}{|c|c|c|c|c|c|c|c|c|c|c|c|c|}
\hline \multirow[b]{2}{*}{ Case No. } & \multirow[b]{2}{*}{ Age (yrs)/Sex } & \multirow[b]{2}{*}{ Diagnosis } & \multirow[b]{2}{*}{ FA } & \multirow[b]{2}{*}{ PA } & \multicolumn{2}{|r|}{ ROI } & \multicolumn{2}{|c|}{ iCBF } & \multirow[b]{2}{*}{$\triangle \mathrm{iCBF}$} & \multirow[b]{2}{*}{$\Delta \% \mathrm{iCBF}$} & \multirow[b]{2}{*}{ MEP } & \multirow[b]{2}{*}{ Postop DWI } \\
\hline & & & & & ROI No. & No. of Findings per ROI & Pre-I & Post-I & & & & \\
\hline 1 & $3 / F$ & EVN & ND & ND & 0 & 0 & - & - & - & - & NR & No \\
\hline 2 & $61 / \mathrm{M}$ & A & $D$ & ND & 1 & 1 & 10.3 & 8.1 & 2.2 & 21.4 & NR & No \\
\hline \multirow[t]{3}{*}{3} & \multirow[t]{3}{*}{$68 / \mathrm{M}$} & \multirow[t]{3}{*}{ GBM } & \multirow[t]{3}{*}{$\mathrm{D}$} & \multirow[t]{3}{*}{ ND } & 2 & 1 & 17.2 & 15.1 & 2.1 & 12.2 & \multirow[t]{3}{*}{ NR } & \multirow[t]{3}{*}{ No } \\
\hline & & & & & 3 & 2 & 13.8 & 10.9 & 2.9 & 21.0 & & \\
\hline & & & & & 4 & 3 & 19.3 & 17.1 & 2.2 & 11.40 & & \\
\hline \multirow[t]{2}{*}{4} & \multirow[t]{2}{*}{$75 / M$} & \multirow[t]{2}{*}{ Meta } & \multirow[t]{2}{*}{$\mathrm{D}$} & \multirow[t]{2}{*}{ ND } & 5 & 1 & 27.5 & 25.8 & 1.7 & 6.2 & \multirow[t]{2}{*}{ NA } & \multirow[t]{2}{*}{ No } \\
\hline & & & & & 6 & 2 & 32.8 & 27.8 & 5 & 15.2 & & \\
\hline \multirow[t]{3}{*}{5} & \multirow[t]{3}{*}{$74 / F$} & \multirow[t]{3}{*}{ Meta } & \multirow[t]{3}{*}{$D$} & \multirow[t]{3}{*}{$D$} & 7 & 1 & 52.4 & 48.3 & 4.1 & 7.8 & NR & No \\
\hline & & & & & 8 & 2 & 41.8 & 39.5 & 2.3 & 5.5 & & \\
\hline & & & & & 9 & 3 & 43.1 & 31.8 & 11.3 & 26.2 & & \\
\hline 6 & $71 / \mathrm{M}$ & GBM & $D$ & ND & 10 & 1 & 33.2 & 29 & 4.2 & 12.7 & NR & No \\
\hline & & & & & 11 & 2 & 45.2 & 31.8 & 13.4 & 29.7 & & \\
\hline 7 & $52 / F$ & GBM & $D$ & ND & 12 & 1 & 42.6 & 36.1 & 6.5 & 15.3 & NR & Yes \\
\hline & & & & & 13 & 2 & 46.1 & 44.2 & 1.9 & 4.1 & & \\
\hline 8 & $35 / M$ & AVM & $D$ & $D$ & 14 & 1 & 22.3 & 5.6 & 16.7 & 74.9 & NR & No \\
\hline & & & & & 15 & 2 & 22.8 & 11.2 & 16.5 & 50.1 & & \\
\hline & & & ND & $D$ & 16 & 3 & 25.9 & 13.3 & 12.6 & 48.6 & & \\
\hline 9 & $10 / \mathrm{M}$ & AVM & $D$ & ND & 17 & 1 & 11.9 & 10.8 & 1.1 & 9.2 & NR & No \\
\hline 10 & $33 / F$ & AVM & $D$ & ND & 18 & 1 & 20.2 & 17.9 & 2.3 & 11.4 & NR & No \\
\hline 11 & $6 / \mathrm{M}$ & AVM & $D$ & ND & 19 & 1 & 101.5 & 94.8 & 6.7 & 6.6 & NR & No \\
\hline 12 & $32 / M$ & AVM & ND & $D$ & 20 & 1 & 38.2 & 4.5 & 33.7 & 88.2 & NR & No \\
\hline & & AN & & & 21 & 2 & 72.3 & 16.7 & 55.6 & 76.9 & & \\
\hline Mean & & & & & & & 35.3 & 25.7 & 9.8 & 26.4 & & \\
\hline
\end{tabular}

$\mathrm{A}=$ astrocytoma; $\mathrm{AN}=$ aneurysm; $\mathrm{D}=$ detected; $\mathrm{EVN}=$ extraventricular neurocytoma; $\mathrm{GBM}=$ glioblastoma; MEP = motor evoked potential; meta = metastatic brain tumor; $N A=$ not available; $N D=$ not detected; $N R=$ no response; $D W I$ = diffusion-weighted image; Pre- $i=$ pre-interruption; Post-i = post-interruption.

* The overall patient population consisted of 8 FA, 2 PA, and 2 FA+PA patients.

a PA is spared by complete lesional resection. For FA+PA, the main trunk of the artery is spared with treatment of the branching small arteries that feed the planned resection area. This procedure was designed to avoid postoperative and unexpected ischemic complications.

\section{Statistical Analysis}

Differences in iCBF between pre- and post-interruption of an artery were evaluated using the paired t-test since these were parametric data. Analyses were conducted using StatFlex software (Artech).

\section{Results}

Color-coded visualization of $\mathrm{CBF}$ in the surgical field and $\mathrm{CBF}$ measurements in the ROIs were obtained in real time with excellent spatiotemporal resolution. ${ }^{13,22}$ In 11 patients (92\%) and 21 ROIs, a clear decline in iCBF was observed after temporary interruption of the lesion-related artery (Table 1). The FA, PA, and FA+PA types were detected in 8,2 , and 2 patients, respectively. The average reduction in $\mathrm{iCBF}$ was $26.4 \% \pm 25.8 \%$ (range $4.1 \%-88.2 \%$ ), and this change was significant (Fig. 2).

In 1 patient (Case 1), we were unable to detect an FA or PA. An artery was resected during removal of the tumor

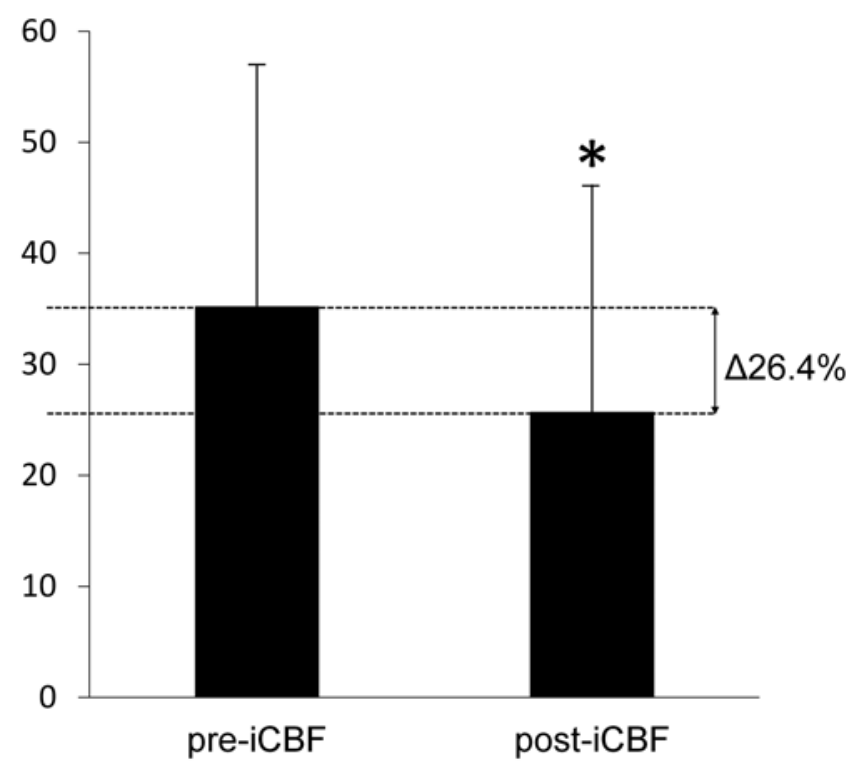

FIG. 2. Fluctuations in iCBF before and after interruption of the lesionrelated arteries. ${ }^{*} p<0.01$ by the paired t-test. 

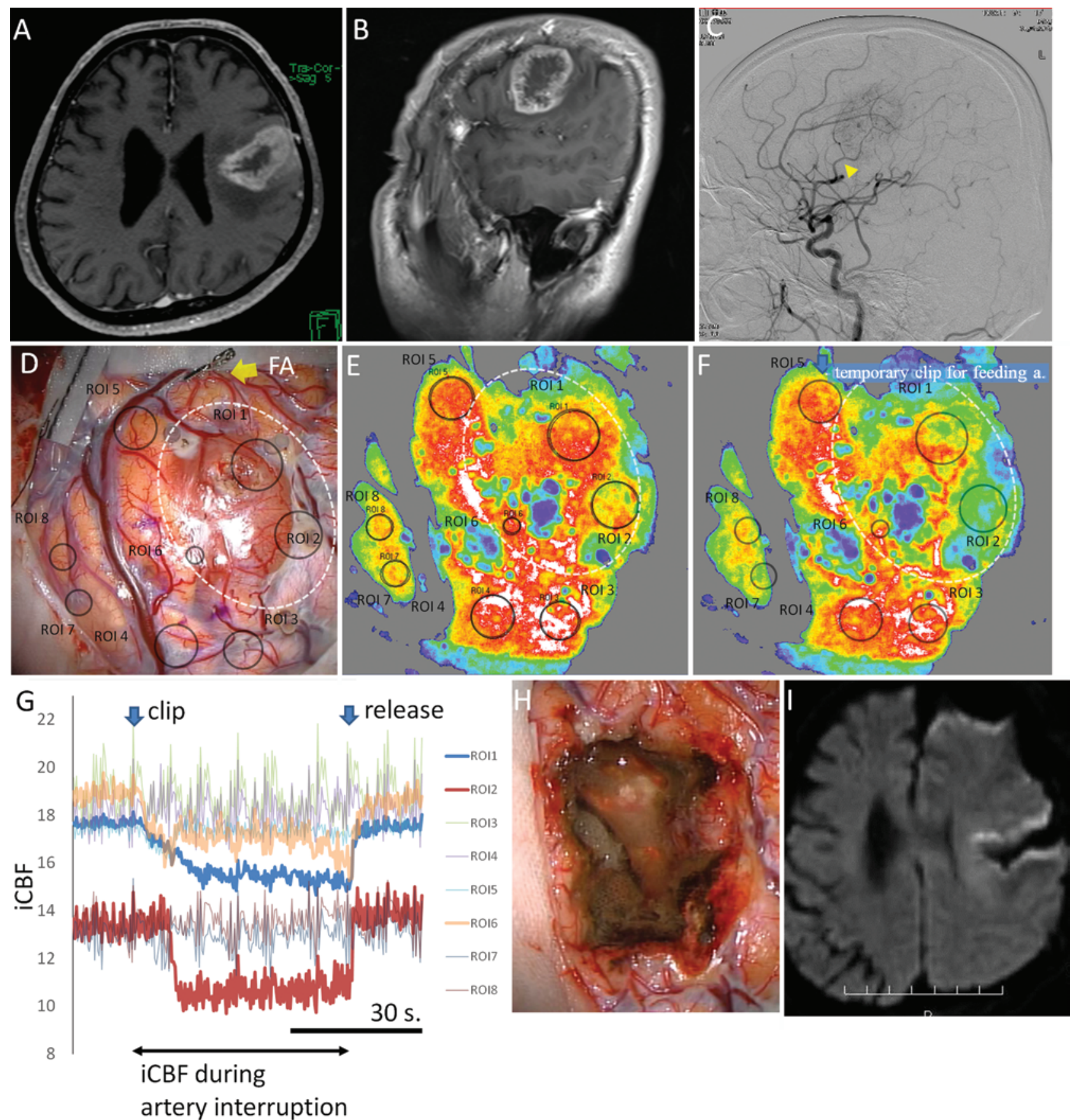

FIG. 3. Case 3. Representative case of a glioblastoma showing an FA. Axial (A) and sagittal (B) views on contrast-enhanced T1-weighted MRI showing a mass lesion in the left premotor area. C: DSA revealing tumor staining that was fed by a frontal branch of the middle cerebral artery (yellow arrowhead). D: Intraoperative photograph showing an FA (yellow arrow) and the planned resection area (white dotted circle). Speckle-pattern imaging before $(E)$ and after $(F)$ temporary interruption of the feeding artery. G: Graph showing the timeline of the iCBF in 8 ROIs. "Clip" indicates the point of interruption of the artery, and "release" indicates the point of reflow after clip release. After clipping, there was an immediate and marked decline in iCBF in ROIs 1, 2, and 6 by $11.4 \%$ to $21.0 \%$, which was maintained during the entire occlusion period of about 45 seconds. Removal of the clip immediately restored baseline perfusion to the pre-interruption level. From this result, the artery was classified as FA (Fig. 1C). The horizontal bar indicates 30 seconds. H: Intraoperative photograph obtained after complete removal of the tumor. I: Diffusion-weighted MR image showing no ischemic complication.

bulk, but no ischemic complications were found on postoperative diffusion-weighted imaging. No case showed a decline in response on motor evoked potential monitoring, which was performed in 11 patients, and there was no speech abnormality on language mapping in awake surgery during interruption of the lesion-related artery (Table 1). One patient (Case 7) had a postoperative ischemic lesion on the posterior side of the right temporal lobe. This may 



FIG. 4. Case 8. Representative case of an AVM showing a PA and FA+PA. A: Susceptibility-weighted MRI showing cerebral hemorrhage in the right temporal lobe. B: DSA revealed a nidus fed by one of the temporal branches of the middle cerebral artery (yellow arrowhead). C: Intraoperative photograph showing the FA+PA (yellow arrow), PA (green arrow), and planned resection area (white dotted circle). D: Speckle-pattern imaging obtained before temporary interruption of arteries. E: Photograph of the application of a temporary clip to the PA. F: Speckle-pattern imaging obtained after temporary interruption of the PA. G: Photograph of the application of a temporary clip to the FA+PA. H: Speckle-pattern imaging after temporary interruption of the FA+PA. I: Timeline of the iCBF in 5 ROls. Clips 1 and 2 indicate the points of interruption of the PA (E and F) and FA+PA (G and $\mathrm{H})$, respectively. Release indicates the point of reflow after clip release. After applying the clip to the artery (C, green arrow), there was an immediate and marked decline in $\mathrm{ICBF}$ in ROI 3 by $48.6 \%$, which was maintained during the entire occlusion period of about 30 seconds. Removal of the clip immediately restored baseline perfusion to the pre-interruption level. From this result, the artery was classified as a PA (Fig. 1D). Next, after applying the clip to the artery (C, yellow arrow), there was an immediate and marked decline in iCBF in ROI 5 (resection planning area) and ROI 1 (surrounding area) by $50.1 \%$ to $74.9 \%$, which was maintained during the entire occlusion period of about 15 seconds. Removal of the clip immediately restored baseline perfusion to the preinterruption level. From this result, the artery was classified as FA+PA (Fig. 1E). J: Intraoperative photograph obtained after the complete removal of an AVM showing spared arteries. K: Diffusion-weighted MR image showing no ischemic complications.

have occurred because we cut one of the temporal branches from the right posterior cerebral artery during surgery for glioblastoma on the reverse side of the temporal lobe.

In a glioblastoma case (Case 3), gadolinium-enhanced MRI showed an irregular ring-enhancing mass lesion in the left frontal lobe facing the motor cortex (Fig. 3A and B). Left carotid angiography revealed a tumor fed by one of the frontal branches of the middle cerebral artery (yellow arrowhead; Fig. 3C). iCBF surface imaging during pre- and post-temporary interruption of the artery revealed an FA that resulted from a decrease in iCBF (ROIs 1,2 , and 6 ) by $11.4 \%$ to $21.0 \%$ in the resection area (white dotted circle) without a change in the surrounding brain surface (Fig. 3D-G). Total resection was achieved without ischemic complications (Fig. $3 \mathrm{H}$ and I).

In Case 8 an AVM occurred with intraparenchymal hemorrhage in the right temporal lobe (Fig. 4A). Left carotid angiography revealed nidus formation that was fed by the temporal branch of the right middle cerebral artery with draining toward the vein of Labbé (Fig. 4B). iCBF surface images obtained during both pre- and post-temporary interruption revealed a PA during the temporary clipping of the artery (green arrow, Fig. 4C) based on a decrease of $\mathrm{iCBF}$ in ROI 3 by $48.6 \%$ in the surrounding area without a change in the resection area (white dotted circle) (Fig. 4D-F and I, Clip 1). In contrast, iCBF surface images 
revealed an FA+PA in the temporary clipping of the other artery (yellow arrow, Fig. 4C) based on a decrease of iCBF in ROI 5 in the resection planning area and ROI 1 in the surrounding area by $50.1 \%$ to $74.9 \%$ (Fig. $4 \mathrm{D}, \mathrm{G}, \mathrm{H}$, and I, Clip 2). The AVM nidus was resected with sparing of these arteries, based on the classification, and treatment of the small branches from the arteries (Fig. 3J). A postoperative diffusion-weighted image showed no ischemic complications (Fig. 4K).

\section{Discussion}

The results of this study indicate the usefulness of LSFI as a noninvasive technique for the intraoperative assessment of microcirculatory flow and CBF alterations related to the planned resection area on the brain surface. Live LSFI images of iCBF were obtained for all 12 patients undergoing direct resection, and LSFI in 11 patients displayed a dynamic response for the sensitive detection of iCBF fluctuations at a high spatial resolution at both preand post-interruption of a lesion-related artery. Based on the iCBF fluctuation pattern, we were able to interpret the cortical vasculature adjacent to the lesion and subsequently resect the tumor bulk or AVM nidus with the appropriate management of the related arteries.

Detecting an FA in the resection area during an early stage of surgery and the prompt application of a temporary clip shortened the operation time by reducing unnecessary bleeding and hemostasis, thereby reducing the physical burden on the patient. If a PA is detected, ischemic complications can be avoided by preserving this artery. Avoiding postoperative neurological deficit requires neurophysiological monitoring, awake surgery, and other methods (i.e., a multimodal approach) to identify the locations of neurological functions and the cortical and subcortical vasculature. ${ }^{12}$ A neurological deficit can thus be avoided by detecting a functional area using this approach, including motor evoked potential response, after temporary interruption of a lesion-related artery. However, if the resection area includes a nonfunctional area, the vasculature related to the lesion remains uncertain because the microcirculation and perfusion area of the lesion-related arteries cannot be visualized. In this situation, LSFI is likely to be useful as a complementary procedure for evaluating lesion-related vasculature and thus avoiding ischemic complications that might cause a neurological deficit.

Several approaches for intraoperative CBF monitoring have been described, primarily for angiographic visualization of the cerebral vasculature or invasive assessment of CBF. DSA remains the gold standard, ${ }^{3,5,28,32}$ but the practical use of this method is limited due to its invasiveness and high cost. Laser Doppler flowmetry is an excellent alternative for instantaneous, continuous, and real-time measurement of relative $\mathrm{CBF}$ fluctuations for determining responses to therapeutic interventions and detecting possible ischemic insults..$^{21}$ Laser Doppler flowmetry has a high temporal resolution and dynamic features but low spatial resolution that impedes blood flow measurements over a wide surface area, including the resection and surrounding areas.

Noninvasive visualization of brain vasculature through ICG videoangiography ${ }^{25,30,31}$ permits inexpensive, highquality imaging of the cortical vasculature. The primary advantage of ICG angiography is the identification of the feeding and draining vessels, while intraluminal perfusion has been measured in recent studies based on the transit time of the dye ${ }^{15,29}$ however, this method does not always give an accurate representation of blood flow. Quantitative information is only obtained during the initial wash-in of the dye (5-10 seconds), multiple injections are not always feasible, and adverse reactions to the dye may occur.. ${ }^{23}$ The plasma half-life of ICG is 3 to 4 minutes, with almost complete clearance after 10 minutes. ${ }^{25}$ To accurately evaluate vessels, repeated temporary interruption of the related arteries is required, and waiting for 2 or more ICG wash-outs from the plasma in order to acquire accurate blood flow data is time consuming. Thus, ICG videoangiography is not suitable for detecting FA or PA based on iCBF fluctuations after several temporary interruptions of the arteries.

Ischemic complications after surgery developed in 1 of our cases due to occlusion of the temporal branch of the posterior cerebral artery, which circulated from the reverse side of the lesion. LSFI was not able to visualize this artery because the method provides information only for the surface of the brain that is exposed in the surgical field. Information about $\mathrm{CBF}$ at the reverse side of the cortex and deep white matter cannot be measured using LSFI, and thus we were unable to detect $\mathrm{CBF}$ decline during surgery in this patient. The combined use of LSFI with methods such as electrophysiology and awake surgery, which can detect injury including ischemia in the deep functional parenchymal region, may be required to avoid all ischemic complications. A single monitoring procedure cannot reveal all $\mathrm{CBF}$ changes, and these methods should be used in combination during surgery.

\section{Conclusions}

Real-time LSFI measurements of intraoperative CBF fluctuations provide information on the arteries related to the planned surgical resection area with high spatial resolution, minimal effort, and the use a repeatable maneuver in a short time. This allows the classification and appropriate management of the arteries and subsequent avoidance of postoperative ischemic complications. Therefore, LSFI reduces the burden on patients and has a cost benefit. This vasculature information indicated on LSFI can be used to preserve neurological function as a procedure complementary to neurophysiological monitoring.

\section{Acknowledgments}

This work was supported by a Grant-in-Aid for Young Scientists from the Japan Society for the Promotion of Science (JSPS) (B) (grant no. 26870408).

\section{References}

1. Akamatsu Y, Shimizu H, Saito A, Fujimura M, Tominaga T: Consistent focal cerebral ischemia without posterior cerebral artery occlusion and its real-time monitoring in an intraluminal suture model in mice. J Neurosurg 116:657-664, 2012

2. Ayata C, Shin HK, Salomone S, Ozdemir-Gursoy Y, Boas DA, Dunn AK, et al: Pronounced hypoperfusion during spreading depression in mouse cortex. J Cereb Blood Flow Metab 24:1172-1182, 2004

3. Barrow DL, Boyer KL, Joseph GJ: Intraoperative angiogra- 
phy in the management of neurovascular disorders. Neurosurgery 30:153-159, 1992

4. Briers JD, Fercher AF: Retinal blood-flow visualization by means of laser speckle photography. Invest Ophthalmol Vis Sci 22:255-259, 1982

5. Chiang VL, Gailloud P, Murphy KJ, Rigamonti D, Tamargo RJ: Routine intraoperative angiography during aneurysm surgery. J Neurosurg 96:988-992, 2002

6. Devor A, Ulbert I, Dunn AK, Narayanan SN, Jones SR, Andermann ML, et al: Coupling of the cortical hemodynamic response to cortical and thalamic neuronal activity. Proc Natl Acad Sci U S A 102:3822-3827, 2005

7. Domoki F, Zölei D, Oláh O, Tóth-Szuki V, Hopp B, Bari F, et al: Evaluation of laser-speckle contrast image analysis techniques in the cortical microcirculation of piglets. Microvasc Res 83:311-317, 2012

8. Dunn AK, Bolay H, Moskowitz MA, Boas DA: Dynamic imaging of cerebral blood flow using laser speckle. J Cereb Blood Flow Metab 21:195-201, 2001

9. Dunn AK, Devor A, Bolay H, Andermann ML, Moskowitz MA, Dale AM, et al: Simultaneous imaging of total cerebral hemoglobin concentration, oxygenation, and blood flow during functional activation. Opt Lett 28:28-30, 2003

10. Dunn AK, Devor A, Dale AM, Boas DA: Spatial extent of oxygen metabolism and hemodynamic changes during functional activation of the rat somatosensory cortex. Neuroimage 27:279-290, 2005

11. Fercher AF, Briers JD: Flow visualization by means of single-exposure speckle photography. Opt Commun 37:326330,1981

12. Gempt J, Förschler A, Buchmann N, Pape H, Ryang YM, Krieg SM, et al: Postoperative ischemic changes following resection of newly diagnosed and recurrent gliomas and their clinical relevance. J Neurosurg 118:801-808, 2013

13. Hecht N, Woitzik J, Dreier JP, Vajkoczy P: Intraoperative monitoring of cerebral blood flow by laser speckle contrast analysis. Neurosurg Focus 27(4):E11, 2009

14. Jones PB, Shin HK, Boas DA, Hyman BT, Moskowitz MA, Ayata C, et al: Simultaneous multispectral reflectance imaging and laser speckle flowmetry of cerebral blood flow and oxygen metabolism in focal cerebral ischemia. J Biomed Opt 13:044007, 2008

15. Kamp MA, Slotty P, Turowski B, Etminan N, Steiger HJ, Hänggi D, et al: Microscope-integrated quantitative analysis of intraoperative indocyanine green fluorescence angiography for blood flow assessment: first experience in 30 patients. Neurosurgery 70 (1 Suppl Operative):65-74, 2012

16. Klijn E, Hulscher HC, Balvers RK, Holland WP, Bakker J, Vincent AJ, et al: Laser speckle imaging identification of increases in cortical microcirculatory blood flow induced by motor activity during awake craniotomy. J Neurosurg 118:280-286, 2013

17. Li M, Miao P, Yu J, Qiu Y, Zhu Y, Tong S: Influences of hypothermia on the cortical blood supply by laser speckle imaging. IEEE Trans Neural Syst Rehabil Eng 17:128-134, 2009

18. Miao P, Li M, Fontenelle H, Bezerianos A, Qiu Y, Tong S: Imaging the cerebral blood flow with enhanced laser speckle contrast analysis (eLASCA) by monotonic point transformation. IEEE Trans Biomed Eng 56:1127-1133, 2009

19. Miao P, Li N, Thakor NV, Tong S: Random process estimator for laser speckle imaging of cerebral blood flow. Opt Express 18:218-236, 2010

20. Miao P, Rege A, Li N, Thakor NV, Tong S: High resolution cerebral blood flow imaging by registered laser speckle contrast analysis. IEEE Trans Biomed Eng 57:1152-1157, 2010

21. Nakagawa A, Hirano T, Uenohara H, Sato M, Kusaka Y, Shirane R, et al: Intraoperative thermal artery imaging of an EC-IC bypass in beagles with infrared camera with detectable wave-length band of 7-14 microm: possibilities as novel blood flow monitoring system. Minim Invasive Neurosurg 46:231-234, 2003

22. Nomura S, Inoue T, Ishihara H, Koizumi H, Suehiro E, Oka F, et al: Reliability of laser speckle flow imaging for intraoperative monitoring of cerebral blood flow during cerebrovascular surgery: comparison with cerebral blood flow measurement by single photon emission computed tomography. World Neurosurg 82:e753-e757, 2014

23. Owens SL: Indocyanine green angiography. Br J Ophthalmol 80:263-266, 1996

24. Paul JS, Luft AR, Yew E, Sheu FS: Imaging the development of an ischemic core following photochemically induced cortical infarction in rats using Laser Speckle Contrast Analysis (LASCA). Neuroimage 29:38-45, 2006

25. Raabe A, Beck J, Gerlach R, Zimmermann M, Seifert V: Near-infrared indocyanine green video angiography: a new method for intraoperative assessment of vascular flow. Neurosurgery 52:132-139, 2003

26. Royl G, Leithner C, Sellien H, Müller JP, Megow D, Offenhauser N, et al: Functional imaging with laser speckle contrast analysis: vascular compartment analysis and correlation with laser Doppler flowmetry and somatosensory evoked potentials. Brain Res 1121:95-103, 2006

27. Sakadzić S, Yuan S, Dilekoz E, Ruvinskaya S, Vinogradov SA, Ayata C, et al: Simultaneous imaging of cerebral partial pressure of oxygen and blood flow during functional activation and cortical spreading depression. Appl Opt 48:D169-D177, 2009

28. Tang G, Cawley CM, Dion JE, Barrow DL: Intraoperative angiography during aneurysm surgery: a prospective evaluation of efficacy. J Neurosurg 96:993-999, 2002

29. Towle EL, Richards LM, Kazmi SM, Fox DJ, Dunn AK: Comparison of indocyanine green angiography and laser speckle contrast imaging for the assessment of vasculature perfusion. Neurosurgery 71:1023-1031, 2012

30. Woitzik J, Horn P, Vajkoczy P, Schmiedek P: Intraoperative control of extracranial-intracranial bypass patency by nearinfrared indocyanine green videoangiography. J Neurosurg 102:692-698, 2005

31. Woitzik J, Peña-Tapia PG, Schneider UC, Vajkoczy P, Thomé $\mathrm{C}$ : Cortical perfusion measurement by indocyanine-green videoangiography in patients undergoing hemicraniectomy for malignant stroke. Stroke 37:1549-1551, 2006

32. Yanaka K, Fujita K, Noguchi S, Matsumaru Y, Asakawa H, Anno I, et al: Intraoperative angiographic assessment of graft patency during extracranial-intracranial bypass procedures. Neurol Med Chir (Tokyo) 43:509-513, 2003

33. Yu J, Miao P, Li M, Qiu Y, Zhu Y, Tong S: High resolution mapping of cortical blood flow by mosaicing the laser speckle images. Conf Proc IEEE Eng Med Biol Soc 2008:37433746, 2008

\section{Disclosure}

The authors report no conflict of interest concerning the materials or methods used in this study or the findings specified in this paper.

\section{Author Contributions}

Conception and design: Ideguchi. Acquisition of data: all authors. Analysis and interpretation of data: Ideguchi. Drafting the article: Ideguchi. Critically revising the article: Ideguchi. Reviewed submitted version of manuscript: Ideguchi. Approved the final version of the manuscript on behalf of all authors: Ideguchi.

\section{Correspondence}

Makoto Ideguchi, Department of Neurosurgery, Yamaguchi University School of Medicine, 1-1-1 Minami-kogushi, Ube, Yamaguchi 755-8505, Japan. email: ideguchi@yamaguchi-u.ac.jp. 